\title{
Expiratory and inspiratory chest computed tomography and pulmonary function tests in cigarette smokers
}

\author{
K. Kubo*, S. Eda*, H. Yamamoto*, K. Fujimoto*, Y. Matsuzawa*, Y. Murayama**, M. Hasegawa**, \\ S. Sone**, F. Sakai
}

Expiratory and inspiratory chest computed tomography and pulmonary function tests in cigarette smokers. K. Kubo, S. Eda, H. Yamamoto, K. Fujimoto, Y. Matsuzawa, Y. Maruyama, M. Hasegawa, S. Sone, F. Sakai. C ERS Journals Ltd 1999.

ABSTRACT: This study evaluated small airway dysfunction and emphysematous destruction of lung parenchyma in cigarette smokers, using chest expiratory highresolution computed tomography (HRCT) and pulmonary function tests (PFT).

The degree of emphysematous destruction was classified by visual scoring (VS) and the average HRCT number at full expiration/full inspiration (E/I ratio) calculated in 63 male smokers and 10 male nonsmokers (group A). The Brinkman smoking index (BI), defined as cigarettes.day ${ }^{-1} \times$ yrs, was estimated. Sixty-three smokers were divided into three groups by PFT: group B1 $(n=7)$, with normal PFT; group B2 $(n=21)$, with diffusing capacity of the lung for carbon monoxide $(D L, C O) \geq 80 \%$ predicted, forced expiratory volume in one second (FEV1) $<\mathbf{8 0 \%}$ pred and/or residual volume (RV) $>120 \%$ pred; and group $B 3(n=35)$, with $D$ L,CO $<80 \%$ pred, FEV $1<80 \%$ pred and/or RV $>120 \%$ pred.

Heavy smokers $(B I \geq 600)(n=48)$ showed a significant increase in emphysema by both VS and E/I. E/I was significantly elevated in both group B2 (mean $\pm \mathrm{SD} 0.95 \pm 0.05)$ and $B 3(0.96 \pm 0.06)$ compared with group $B 1(0.89 \pm 0.03)$. VS could not differentiate group $B 2(3.9 \pm 5.0)$ from $B 1(1.1 \pm 1.6)$.

These findings suggest that the expiration/inspiration ratio reflects hyperinflation and airway obstruction, regardless of the functional characteristics of emphysema, in cigarette smokers.

Eur Respir J 1999; 13: 252-256.
Depts of *Medicine and **Radiology, Shinsu University Schhol of Medicine, Matsumoto, Japan. +Dept of Radiology, Tokyo Women's Medical College, Tokyo, Japan.

Correspondence: K. Kubo

First Dept of Internal Medicine

Shinshu University School of Medicine

3-1-1 Asahi

Matsumoto, 390-8621

Japan

Fax: 81263363722

Keywords: Cigarette smokers expiratory computed tomography pulmonary function tests visual score

Received: March 91998

Accepted after revision October 81998

This work was in part supported by a Grant-in-Aid for Scientific Research (B), no. 08457179, from the Ministry of Education, Science, Sports and Culture of Japan.
Cigarette smoking is the main cause of emphysema [13]. Emphysema is classified into three main subtypes, centrilobular, panlobular and paraseptal, on the basis of the predominant location of the lung destruction $[1,2]$. In Japan panlobular emphysema, associated with $\alpha_{1}$-protease inhibitor deficiency, is rare [4] and centrilobular emphysema, associated with cigarette smoking, is the most common form. Cigarette smoking produces inflammatory changes in the smallest airways $[5,6]$, especially respiratory bronchioles (respiratory bronchiolitis). This pathological process leads to dilatation and destruction of the small airways, characterized as emphysema of centrilobular type [7]. Pulmonary function tests (PFT) [8] and chest radiological examination, including plain chest radiographs and chest computed tomography (CT) [9-11], are the principal methods used to detect emphysema. However, these conventional methods are not sensitive enough to detect earlier morphological and functional abnormalities of the small airways.

Some recent reports have indicated that CT scans obtained at full expiration show areas of emphysema and air trapping with great clarity and may, therefore, allow the early detection of chronic obstructive pulmonary disease (COPD) or emphysema [7, 12-15]. A previous study by the authors [16] demonstrated that the average ratio of high-resolution CT (HRCT) at full expiration to that at full inspiration (E/I ratio) reflects the air trapping in the small airways in emphysema, whereas the degree of emphysema estimated by visual observation on HRCT scanned at full inspiration visual score (VS) may reflect the emphysematous destruction of lung parenchyma. $\mathrm{E} / \mathrm{I}$ is easy to obtain in a single breath-hold using a helical (spiral) CT scan and is quantitative. To determine whether the ratio is useful in detecting earlier changes in small airway dysfunction and emphysematous destruction of lung parenchyma in cigarette smoking, E/I was compared with VS and the results of PFT in cigarette smokers and normal, nonsmoking volunteers.

\section{Materials and methods}

\section{Study subjects}

Sixty-three consecutive male current smokers or exsmokers who consulted the Shinshu University Hospital between April 1994 and September 1997, with complaints of cough, sputum or shortness of breath were studied, along with 10 normal nonsmoking volunteers (table 1). 
Table 1. - Profiles of subjects

\begin{tabular}{lcc}
\hline & $\begin{array}{c}\text { Nonsmokers } \\
\text { (group A) }\end{array}$ & $\begin{array}{c}\text { Cigarette } \\
\text { smokers }\end{array}$ \\
\hline $\mathrm{n}$ & 10 & 63 \\
Age yrs & $60.8 \pm 10.9$ & $63.0 \pm 10.9$ \\
Body height cm & $165 \pm 2$ & $165 \pm 7$ \\
Body weight kg & $63.3 \pm 7.1$ & $59.0 \pm 9.1$ \\
BI & 0 & $1010 \pm 661^{*}$ \\
VC \% pred & $108.4 \pm 23.9$ & $101.4 \pm 22.0$ \\
FEV1 \% pred & $109.5 \pm 19.0$ & $67.2 \pm 36.0^{*}$ \\
FEV1/FEV \% & $85.4 \pm 5.7$ & $53.5 \pm 18.6^{*}$ \\
RV/TLC \% & $42.6 \pm 14.5$ & $53.0 \pm 12.4^{*}$ \\
RV \% pred & $116.1 \pm 34.5$ & $153.1 \pm 43.4^{*}$ \\
$D L, C O \%$ pred & $106.8 \pm 16.7$ & $74.9 \pm 33.6^{*}$ \\
$D L, C O / V$ A \% pred & $120.1 \pm 27.4$ & $70.3 \pm 33.0^{*}$ \\
\hline Val ar expresed
\end{tabular}

Values are expressed as means \pm SD. BI: Brinkman smoking index, the number of cigarettes smoked $\cdot$ day $^{-1} \times$ number of years smoked (to convert to pack-yrs, divide by 20); VC: vital capacity; FEV1: forced expiratory volume in one second; FEV: forced expiratory volume; RV: residual volume; TLC: total lung capacit; DL,CO: diffusing capacity of the lung for carbon monoxide; $V \mathrm{~A}$ : alveolar volume. *: ${ }^{*}<0.05$ compared with nonsmokers.

Evaluation included medical history, physical examination, routine laboratory tests, serum $\alpha_{1}$-antitrypsin level, electrocardiogram (ECG), chest radiograph and CT.

Patients with lung diseases other than COPD were excluded. Subjects who clearly satisfied the definition of chronic bronchitis $[1,2]$ and showed dilation of the bronchi on CT images were also excluded.

After the purpose of the study had been explained, each subject gave signed informed consent.

\section{Pulmonary function tests}

Spirometry was performed with a water spirometer (Godart Expirograph; Godart-Statham, Bilthoven, the Netherlands). Vital capacity (VC) and forced expiratory volume in one second (FEV1) were calculated and expressed as a percentage of the predicted value. Functional residual capacity (FRC) was measured by He dilution and total lung capacity (TLC) and residual volume (RV) were calculated from the lung volume. VC, FEV1 and RV were expressed as a percentage of the predicted values using methods described previously [17-19]. The diffusing capacity of the lung for carbon monoxide $(D \mathrm{~L}, \mathrm{CO})$ was measured by the single-breath method (Pulmorecorder, model R1551S; Anima, Tokyo, Japan). The ratio of DL,CO to alveolar volume $(V \mathrm{~A})$ was also calculated and expressed as a percentage of the predicted values. The predicted values for $D \mathrm{~L}, \mathrm{CO}$ and $D \mathrm{~L}, \mathrm{CO} / V \mathrm{~A}$ were determined as described previously $[20,21]$.

\section{Chest high-resolution computed tomography}

A helical CT scanner (HiSpeed Advantage; GE Medical Systems, Milwaukee, USA) was used. After standard 10mm-thick contiguous scanning at full inspiration for screening of chest abnormalities, scanning with 3-mm collimation (scan time of $1 \mathrm{~s}$ ) was performed at both full inspiration and full expiration at the carina (the levels of the upper lung fields) and orifice of the pulmonary veins (the levels of the lower lung fields). HRCT images were reconstructed with the ultra-high-density display kernels. Hard-copy images were photographed using the window setting appropriate for the lungs (level -700 Hounsfield unit (HU); width 1,000 HU).

VS was assessed by the method of GoDDARD et al. [22] by inspection of four HRCT hemislices obtained at full inspiration. Emphysematous destruction was identified as areas of low attenuation and hypovascular regions in the lungs [22-24]. By using a five-point scale, the percentage of the lung involved in emphysematous change was determined as follows: 0 , no emphysema; $1,1-25 \%$ involvement; $2,26-50 \% ; 3,51-75 \%$; and 4, 76-100\%. The total score for each individual was calculated by adding together the points $(0-16)$ for the four hemislices. For E/I the average HRCT number in each lung field was calculated at full inspiratory (TLC level) and expiratory (RV level) positions. The ratio was obtained by dividing the CT number at RV level by that at TLC level. All radiographs, especially VS, were interpreted by an experienced chest radiologist (F. Sakai) who had no knowledge of the clinical, functional or chest radiographic data, because previous studies have shown good intra- and interobserver correlations for the subjective estimation of emphysema $[23,25]$.

\section{Classification of subjects}

Ten nonsmokers (group A) and 63 cigarette smokers were classified according to their cumulative smoking, using the Brinkman smoking index (BI) [16], defined as the number of cigarettes smoked.day ${ }^{-1} \times$ number of years smoked (to convert to pack-yrs, divide by 20) (table 2) and according to PFT results (table 3 ). The subjects with BI $<600(\mathrm{n}=15)$ and $\geq$ BI $600(\mathrm{n}=48)$ are defined arbitrarily as mild and heavy smokers, respectively (table 2). By PFT (table 3), the 63 cigarette smokers were divided into three groups: group B1 $(\mathrm{n}=7)$, with normal PFT; group B2 $(\mathrm{n}=21)$, with (all values as $\%$ predicted) $D \mathrm{~L}, \mathrm{CO} \geq 80 \%$, FEV $1<80 \%$ and/or RV $>120 \%$; and group B3 $(\mathrm{n}=35)$, with $D$ L,CO $<80 \%$, FEV $1<80 \%$ and/or RV $>120 \%$.

Table 2. - Classification of cigarette smokers by the Brinkman smoking index $(\mathrm{BI})$

\begin{tabular}{lcc}
\hline & $\begin{array}{c}\text { Mild smokers } \\
(\mathrm{BI}<600)\end{array}$ & $\begin{array}{c}\text { Heavy smokers } \\
(\mathrm{BI} \geq 600)\end{array}$ \\
\hline $\mathrm{n}$ & 15 & 48 \\
Age yrs & $56.2 \pm 13.1$ & $64.8 \pm 9.6$ \\
Body height cm & $166 \pm 8$ & $165 \pm 7$ \\
Body weight kg & $64.6 \pm 8.6$ & $58.0 \pm 9.1$ \\
BI & $350 \pm 141$ & $1212 \pm 628^{*}$ \\
VC \% pred & $118.2 \pm 18.2$ & $96.8 \pm 21.2$ \\
FEV1 \% pred & $95.1 \pm 32.2$ & $60.8 \pm 34.2^{*}$ \\
FEV1/FEV \% & $69.7 \pm 16.5$ & $48.9 \pm 16.9^{*}$ \\
RV/TLC \% & $47.1 \pm 11.3$ & $55.2 \pm 11.8$ \\
RV \% pred & $134.5 \pm 24.3$ & $159.4 \pm 45.4$ \\
$D L$, CO \% pred & $92.5 \pm 22.5$ & $70.4 \pm 34.9^{*}$ \\
$D$ LCO/VA \% pred & $82.2 \pm 19.5$ & $67.0 \pm 34.9^{*}$ \\
\hline
\end{tabular}

Values are expressed as means \pm SD. For definitions see legend to table $1 .{ }^{*}: \mathrm{p}<0.05$ compared with mild smokers. 
Table 3. - Classification of cigarette smokers by pulmonary function tests (PFT)

\begin{tabular}{lccc}
\hline & Group B1 & Group B2 & Group B3 \\
\hline $\mathrm{n}$ & 7 & 21 & 35 \\
Age yrs & $58.0 \pm 11.5$ & $60.1 \pm 14.4$ & $65.4 \pm 7.6$ \\
Body height cm & $164 \pm 12$ & $165 \pm 8$ & $165 \pm 7$ \\
Body weight kg & $62.4 \pm 12.8$ & $60.5 \pm 8.9$ & $57.5 \pm 8.6$ \\
BI & $697 \pm 285$ & $915 \pm 876$ & $1130 \pm 538^{*}$ \\
VC \% pred & $110.8 \pm 6.6$ & $109.1 \pm 20.9$ & $95.1 \pm 22.6$ \\
FEV1 \% pred & $122.2 \pm 17.5$ & $60.3 \pm 29.6^{*}$ & $53.1 \pm 32.3^{*}$ \\
FEV1/FEV \% & $79.0 \pm 1.8$ & $61.1 \pm 16.7^{*}$ & $44.5 \pm 15.0^{*}$ \\
RV/TLC \% & $35.1 \pm 4.8$ & $51.2 \pm 10.7^{*}$ & $56.6 \pm 11.7^{*}$ \\
RV \% pred & $89.8 \pm 7.3$ & $156.7 \pm 40.9^{*}$ & $159.9 \pm 41.1^{*}$ \\
$D$ L,CO \% pred & $101.0 \pm 20.1$ & $105.2 \pm 19.2$ & $53.0 \pm 24.0^{*}$, \\
$D L$, CO/VA \% pred & $125.0 \pm 23.5$ & $94.0 \pm 18.8$ & $49.8 \pm 22.5^{*}$, \\
\hline Valy a expresed
\end{tabular}

Values are expressed as means \pm SD. Group B1: smokers with normal PFT; group B2: smokers with diffusing capacity for carbon monoxide $(D \mathrm{~L}, \mathrm{CO}) \geq 80 \%$ pred, forced expiratory volume in one second (FEV1) $<80 \%$ pred and/or residual volume (RV) $>120 \%$ pred; group B3: smokers with $D \mathrm{~L}, \mathrm{CO}<80 \%$ pred, FEV $1<80 \%$ pred and/or RV $>120 \%$ pred. BI: Brinkman smoking index, the number of cigarettes smoked $\cdot$ day $^{-1} \times$ number of years smoked (to convert to pack-yrs, divide by 20); VC: vital capacity; FEV: forced expiratory volume; TLC: total lung capacity; VA: alveolar volume. *: $\mathrm{p}<0.05$ compared with group $\mathrm{B} 1{ }^{+}: \mathrm{p}<0.05$ compared with group $\mathrm{B} 2$.

\section{Statistical analysis}

The data in the text, tables and figures are presented as means \pm SD. One-way analysis of variance (ANOVA) and Student's t-test were used to examine the significance of differences between groups. A p-value $<0.05$ was considered statistically significant.

\section{Results}

\section{Subject profiles}

There were no significant differences in age, body height and weight between cigarette smokers and nonsmokers (table 1). As shown in tables 1 and 2, PFT results revealed significant abnormalities in cigarette smokers and heavy smokers compared with nonsmokers and mild smokers, respectively. The BI of group B1 was $697 \pm 285$, which was not significantly different from cigarette smokers in groups B2 and B3 (table 3).

Relationships between expiration/inspiration ratio, visual score smoking and pulmonary function tests

Figures 1 and 2 show the E/I ratio and VS, respectively, of nonsmokers (group A), mild and heavy smokers. Both $\mathrm{E} / \mathrm{I}$ ratio and VS were significantly higher in heavy smokers than in nonsmokers and mild smokers, although not significantly different between nonsmokers and mild smokers. Two mild smokers showed high VS (fig. 2). Their medical history and laboratory data were checked, including serum levels of $\alpha_{1}$-antitrypsin, but these scores could not be explained. It seems likely that these subjects were particularly susceptible to smoking for the development of emphysema.

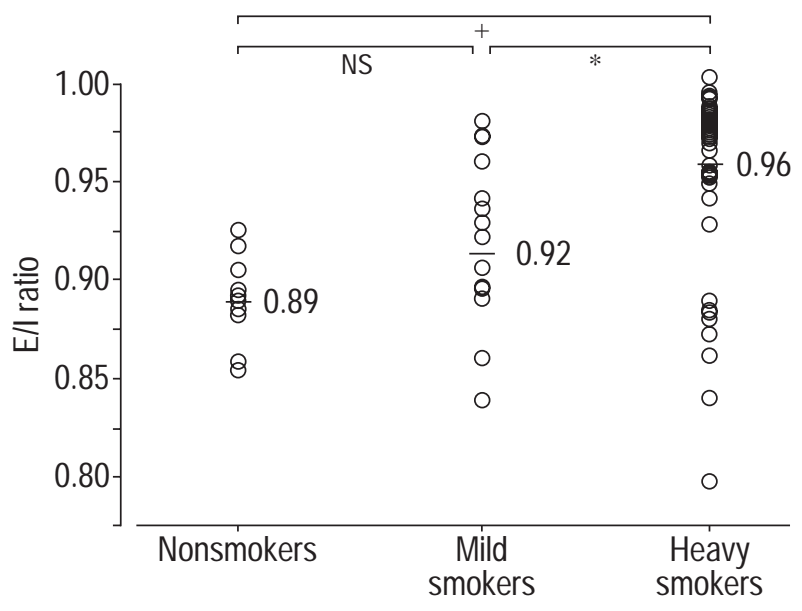

Fig. 1. - Expiration/inspiration (E/I) ratio in nonsmokers $(n=10)$ and mild (Brinkman smoking index $(\mathrm{BI})<600 ; \mathrm{n}=15$ ) and heavy smokers (BI $\geq 600 ; n=48)$. E/I ratio: average number of chest high-resolution computed tomography (HRCT) scanning at full E/full I; BI: number of cigarettes smoked.day ${ }^{-1} \times$ number of years smoked (to convert to pack-yrs, divide by 20). The horizontal bars show mean values of each group. Ns: nonsignificant; ${ }^{*}: \mathrm{p}<0.05 ;^{+}: \mathrm{p}<0.005$.

Figures 3 and 4 show the difference in the E/I and VS, respectively, between groups $\mathrm{A}, \mathrm{B} 1, \mathrm{~B} 2$ and $\mathrm{B} 3$. For the $\mathrm{E} / \mathrm{I}$ ratio, both group $\mathrm{B} 2(0.95 \pm 0.05)$ and group $\mathrm{B} 3$ $(0.96 \pm 0.06)$ had significantly higher values than group A $(0.85 \pm 0.03)$ and group B1 $(0.89 \pm 0.03)$. For VS there was no significant difference between group B1 (1.1 \pm 1.6$)$ and group B2 (3.9 \pm 5.0$)$. The value of VS in group B3 $(9.3 \pm$ 4.1) was significantly higher than that in group B2.

\section{Discussion}

The aim of the present study was to evaluate the changes in small airway dysfunction and emphysematous destruction of lung parenchyma in cigarette smokers using VS of emphysematous destruction and the E/I ratio. Mild smokers $(\mathrm{BI}<600)$ could not be discriminated from nonsmokers by either method, E/I or VS. However, heavy smokers (BI

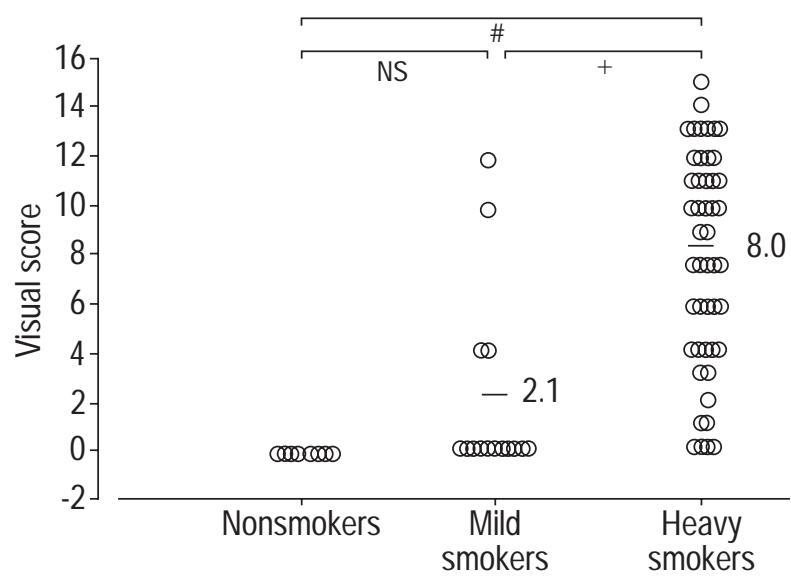

Fig. 2. - Visual score in nonsmokers $(n=10)$ and mild (Brinkman smoking index $(B I)<600 ; n=15)$ and heavy smokers $(B I \geq 600 ; n=48)$. Visual score: visual scoring of emphysematous changes in chest highresolution computed tomography scanning. The horizontal bars show mean values of each group. NS: nonsignificant; +: $\mathrm{p}<0.005 ;{ }^{\#}: \mathrm{p}<0.0005$. 


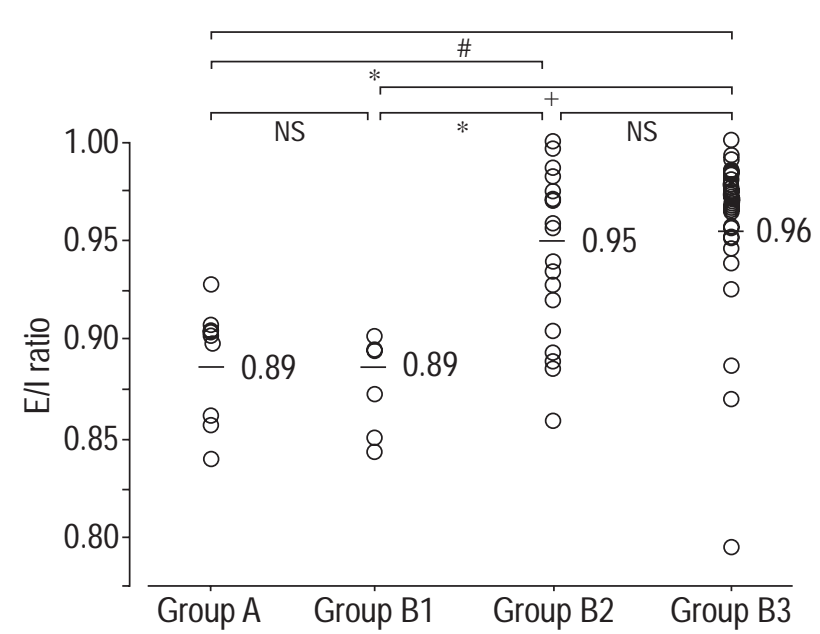

Fig. 3. - Expiration/inspiration (E/I) ratio in nonsmokers (group A; n= 10 ), smokers with normal pulmonary function tests (group B1; $\mathrm{n}=7$ ), smokers with carbon monoxide diffusing capacity $(D \mathrm{~L}, \mathrm{CO}) \geq 80 \%$ pred, forced expiratory volume in one second $(\mathrm{FEV} 1)<80 \%$ pred and/or residual volume (RV) $>120 \%$ pred (group B2; $n=21$ ), and smokers with $\mathrm{DL}, \mathrm{CO}<80 \%$ pred, $\mathrm{FEV} 1<80 \%$ pred and/or RV $>120 \%$ pred (group B3; $\mathrm{n}=35$ ). The horizontal bars show mean values of each group. ${ }^{*}: \mathrm{p}<0.05$; : $\mathrm{p}<0.005 ;{ }^{\#}: \mathrm{p}<0.0005$.

$\geq 600$ ) showed significantly higher values of E/I as well as VS compared with those of nonsmokers and mild smokers.

Several studies have focused on emphysematous changes using chest CT scanning in cigarette smokers. KONDOH et al. [26] studied 35 patients with chronic asthma and found that 18 smokers $(36.7 \pm 15.4$ pack-yrs $)$ had significantly higher visual emphysematous scores on CT scan than did 17 nonsmokers, and that this VS significantly correlated with pack-yrs of cigarette consumption. REMY$\mathrm{J}_{\mathrm{ARDIN}}$ et al. [27] reported that HRCT revealed emphysematous change in $20(21 \%)$ out of 98 smokers with $12.8 \pm 10.41$ pack-yrs, despite normal PFT, and in two $(8 \%)$ out of 26 exsmokers with $11.7 \pm 7.03$ pack-yrs, and no sign of emphysema in 51 nonsmokers. BETSUYAKU et al. [28] found that in 49 asymptomatic smokers, HRCT detected a low attenuation area (LAA) in $20(41 \%)$ subjects and that

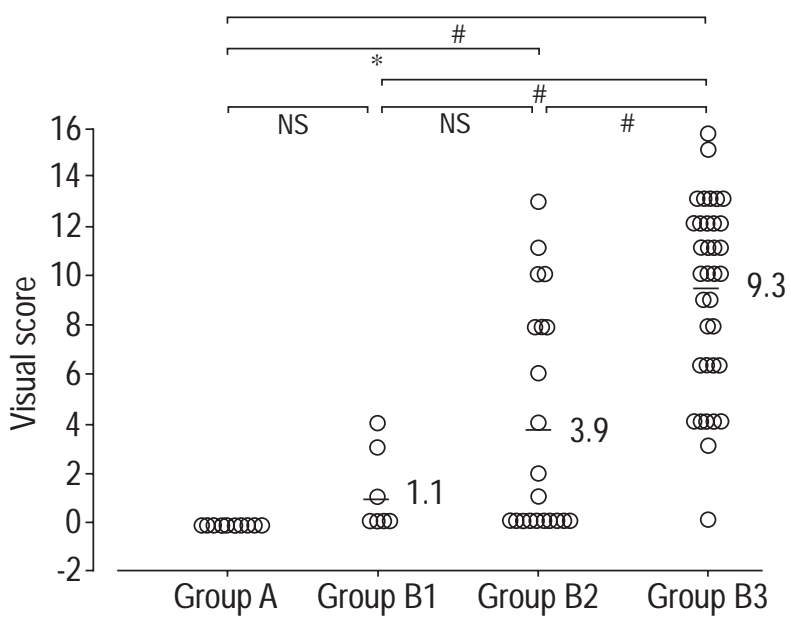

Fig. 4. - Visual score in groups A $(n=10), B 1(n=7), B 2(n=21)$ and B3 $(n=35)$. Visual score: visual scoring of emphysematous changes in chest high-resolution computed tomography scanning. For definitions of groups see legend to figure 3 . The horizontal bars show mean values of each group. ${ }^{*}: \mathrm{p}<0.05 ;{ }^{\#}: \mathrm{p}<0.0005$. these 20 subjects showed significantly lower values of FEV1, DL,CO and $D \mathrm{~L}, \mathrm{CO} / V_{\mathrm{A}}$ compared with 29 subjects without LAA, although all mean values were within normal limits. In the present study there were no significant differences in E/I or VS among nonsmokers, mild smokers and smokers with normal PFT (group B1). Furthermore, no other study of CT or HRCT at full expiration has focused on cigarette smokers.

The classification of subjects by PFT results into groups $\mathrm{B} 2$ and $\mathrm{B} 3$ in the present study was almost identical to that employed by HeREMANS et al. [13], who defined a subgroup of cigarette smokers with $D \mathrm{~L}, \mathrm{CO} \geq 80 \%$ pred consisting of subjects with airway obstruction and hyperinflation and without functional emphysema, and a subgroup with $D$ L,CO $<80 \%$ pred consisting of those with functional emphysema. The former and the latter subgroups are equivalent to the present groups B2 and B3, respectively. $\mathrm{E} / \mathrm{I}$ was significantly higher in both groups $\mathrm{B} 2$ and $\mathrm{B} 3$ than in nonsmokers (group A) and group B1. However, VS did not differentiate group B2 from groups A and B1. These findings suggest that the E/I ratio may detect the airway obstruction and hyperinflation as well as the emphysematous destruction of the lung parenchyma, whereas VS does not detect the airway obstruction and hyperinflation.

The usefulness of E/I is explicable because several studies have revealed that CT or HRCT at full expiration is useful in identifying subtle airway abnormalities and air trapping in the evaluation of emphysema and COPD [1215]. KNUDSON et al. [12] noted that CT scans taken at full expiration revealed abnormal permanent enlargement of small air spaces in emphysema. Heremans et al. [13] demonstrated that lung CT densities at FRC reflected the degree of hyperinflation, i.e. enlargement of distal airways. LAMERS et al. [14] reported that lung CT densities scanned at $10 \%$ of VC differentiated patients with chronic bronchitis from normal controls, but that CT densities at $90 \%$ VC (full inspiration) did not. MINIATI et al. [15] revealed that when the mean HRCT number and percentage area of the lung with low attenuation values $(<-900 \mathrm{HU})$ were examined at full inspiration and full expiration in control subjects and in patients with COPD, only at full expiration was there a significant difference between two groups. The $\mathrm{E} / \mathrm{I}$ ratio used in the present study also reflects air trapping or airflow obstruction [16].

Because the E/I ratio is a quantitative method which can be easily obtained, it could be used to detect emphysema in mass screening programmes and to estimate airflow obstruction in diseases affecting small airways. A recent report [29] revealed the usefulness of the E/I ratio in demonstrating that pulmonary infection with Mycobacterium aviumintracellulare (MAI) is probably a small airway disease, since the small nodules and ectasia of small airways caused by MAI infection are located in centrilobular regions in HRCT and the E/I ratio is significantly increased at the level of affected lesions by MAI.

A previous study [16] showed that VS has a high correlation with $D \mathrm{~L}, \mathrm{CO}$ or $D \mathrm{~L}, \mathrm{CO} / V \mathrm{~A}$, which reflects the degree of emphysematous destruction of the lungs, whereas it shows a low correlation with RV/TLC or RV, which reflects air trapping. Therefore, it seems that VS could differentiate group B2 from group B1.

In summary, the expiration/inspiration ratio and visual score were evaluated using high-resolution computed tomography in cigarette smokers. Smokers were divided by 
the Brinkman smoking index into mild and heavy smokers, and by pulmonary function tests into smokers with normal pulmonary function tests (group B1), smokers with high carbon monoxide diffusing capacity and low forced expiratory volume in one second (group B2) and smokers with low carbon dioxide diffusing capacity and low forced expiratory volume (group B3). Both values were significantly higher in heavy smokers than in nonsmokers and mild smokers. The expiration/inspiration ratio was significantly higher in groups B2 and B3 than in group B1. Visual scoring could not differentiate group B2 from B1, but its value in group B3 was higher than that in group B2. The expiration/inspiration ratio may reflect the hyperinflation and airway obstruction induced by lung emphysematous changes, regardless of the functional characteristics of emphysema, and may be useful in estimating the airflow obstruction and emphysematous destruction of lung parenchyma in cigarette smokers.

\begin{abstract}
Acknowledgements. The authors thank Y. Yamazaki, S. Yamaguchi, K. Okada, S. Yoshikawa, and T. Kobayashi, Dept of Medicine, Shinshu University Hospital, for their help and suggestions.
\end{abstract}

\section{References}

1. Snider GL, Kleinerman J, Thurlbeck WM, Bengali ZH The definition of emphysema. Report of a National Heart, Lung, and Blood Institute, Division of Lung Diseases Workshop. Am Rev Respir Dis 1985; 132: 182-185.

2. Celli BR, Snider GL, Heffner J, et al. ATS statement: standards for the diagnosis and care of patients with chronic obstructive pulmonary disease. Am J Respir Crit Care Med 1995; 152: S77-S120.

3. Sherman CB. The health consequences of cigarette smoking. Med Clin North Am 1992; 76: 355-375.

4. Seyama K, Nukiwa T, Souma S, Shimizu K, Kira S. $\alpha_{1^{-}}$ Antitrypsin-deficient variant Siiyama (Ser53[TCC] to Phe53[TTC]) is prevalent in Japan: status of $\alpha_{1}$-antitrypsin deficiency in Japan. Am J Respir Crit Care Med 1995; 152: 2119-2126.

5. Niewoehner DE, Kleinerman J, Rice DB. Pathologic changes in the peripheral airways of young cigarette smokers. N Engl J Med 1974; 291: 755-758.

6. Hunninghake GW, Gadek JE, Kawanami O, Ferrans VJ, Crystal RG. Inflammatory and immune processes in the human lung in health and disease: evaluation by bronchoalveolar lavage. Am J Pathol 1979; 97: 149-206.

7. Gurney JW. The pathophysiology of airway disease. $J$ Thorac Imaging 1991; 624: 362-364.

8. Burrows B. Early detection of airways obstruction. Chest 1974; 65: 239-240.

9. Sanders C. The radiographic diagnosis of emphysema. Radiol Clin North Am 1991; 19: 1019-1030.

10. Stem EJ, Frank MS. CT of the lung in patients with pulmonary emphysema: diagnosis, quantification, and correlation with pathologic and physiologic findings. $\mathrm{Am}$ J Roentgenol 1994; 162: 791-798.

11. Thurlbeck WM, Muller NL. Emphysema: definition, imaging, and quantification. Am J Roentgenol 1994; 163 : 1017-1025.

12. Knudson RJ, Standen JR, Kaltenborn WT, et al. Expiratory computed tomography for assessment of suspected pulmonary emphysema. Chest 1991; 99: 1357-1366.
13. Heremans A, Verschakelen JA, van Fraeyenhoven L, Demedts M. Measurement of lung density by means of quantitative CT scanning: a study of correlations with pulmonary function tests. Chest 1992; 102: 805-811.

14. Lamers RJ, Thelissen GR, Kessels AG, Wouters EF, van Engelshoven JM. Chronic obstructive pulmonary disease: evaluation with spirometrically controlled CT lung densitometry. Radiology 1994; 193: 109-113.

15. Miniati M, Filippi E, Falashi F, et al. Radiologic evaluation of emphysema in patients with chronic obstructive pulmonary disease. Am J Respir Crit Care Med 1995; 151: 1359-1367.

16. Eda S, Kubo K, Fujimoto K, Matsuzawa Y, Sekiguchi M, Sakai F. The relations between expiratory chest CT using helical CT and pulmonary function tests in emphysema. Am J Respir Crit Care Med 1997; 155: 1290-1294.

17. Baldwin ED, Cournand A, Richards DW Jr. Pulmonary insufficiency. I. Physiological classification, clinical methods of analysis, standard values in normal subjects. Medicine 1948; 27: 243-278.

18. Berglund E, Birath G, Bjure J, et al. Spirometric studies in normal subjects. I. Forced expirograms in subjects between 7 and 70 years of age. Acta Med Scand 1963; 173: 185-191.

19. Grimby G, Soderholm B. Spirometric studies in normal subjects. III. Static lung volumes and maximum voluntary ventilation in adults with a note on physical fitness. Acta Med Scand 1963; 173: 199-206.

20. Nishida O, Kambe M, Sewake N, et al. Pulmonary function in healthy subjects and its prediction. 5. Pulmonary diffusion capacity in adults. Jpn J Clin Pathol 1976; 24: 941-947.

21. Burrows B, Kasik JE, Hiden AH, Barclay WR. Clinical usefulness of the single-breath pulmonary diffusion capacity test. Am Rev Respir Dis 1961; 84: 789-806.

22. Goddard PR, Nicholson EM, Laszlo G, Watt I. Computed tomography in pulmonary emphysema. Clin Radiol 1982; 33: 379-387.

23. Foster WL Jr, Pratt PC, Roggli VL, Godwin JD, Halvorsen RA Jr, Putman CE. Centrilobular emphysema: CTpathologic correlation. Radiology 1986; 159: 27-32.

24. Gurney JW, Jones KK, Robbins RA, et al. Regional distribution of emphysema: correlation of high-resolution CT with pulmonary function tests in unselected smokers. Radiology 1992; 183: 457-463.

25. Morrison NJ, Abboud RT, Ramadan F, et al. Comparison of single breath carbon monoxide diffusing capacity and pressure-volume curve in detecting emphysema. Am Rev Respir Dis 1989; 139: 1179-1187.

26. Kondoh Y, Tanigichi H, Yokoyama S, Taki F, Satake T. Emphysematous change in chronic asthma in relation to cigarette smoking: assessment by computed tomography. Chest 1990; 97: 845-849.

27. Remy-Jardin M, Remy J, Boulenguez C, Sobaszek A, Edme JL, Furon D. Morphologic effects cigarette smoking on airways and pulmonary parenchyma in healthy adult volunteers: CT evaluation and correlation with pulmonary function tests. Radiology 1993; 186: 107-115.

28. Betsuyaku T, Yoshioka A, Nishimura M, Miyamoto K, Kawakami Y. Pulmonary function is diminished in older asymptomatic smokers and exsmokers with low attenuation areas on high-resolution computed tomography. Respiration 1996; 63: 333-338.

29. Kubo K, Yamazaki Y, Masubuchi T, et al. Pulmonary infection with Mycobacterium avium-intracellulare leads to air trapping distal to small airways. Am J Respir Crit Care Med 1998; 158: 979-984. 